\title{
Documentos
}

\section{La preeclampsia un problema de salud pública mundial}

\author{
Víctor Manuel Vargas $H^{1}{ }^{1}$, Gustavo Acosta A. ${ }^{1}$, Mario Adán Moreno E. ${ }^{2}$ \\ ${ }^{1}$ Dirección de Investigación, ${ }^{2}$ Laboratorio de Inmunobiología. Hospital Juárez de México, Secretaría de Salud, México DF.
}

\section{RESUMEN}

La preeclampsia es un problema de salud pública y una de las principales causas de muerte materna. La prevalencia oscila entre 1,8-16,7\%. La causa sigue desconocida y se asocia a problemas de salud importantes, existiendo muchos retos para la predicción, prevención y tratamiento. Las medidas profilácticas como dosis baja de aspirina y suplementos de calcio, requieren mayor evidencia para uso rutinario. El control prenatal, diagnóstico oportuno, manejo adecuado, y parto son las medidas más eficaces para disminuir la tasa de mortalidad por esta causa. Los factores sociales y de atención médica oportuna deben prevalecer en la población, sin embargo, estos son limitados en poblaciones marginadas sin acceso a servicios médicos. Podemos concluir que los sistemas de salud de todas las poblaciones deben identificar y asistir medicamente a las mujeres que están en mayor riesgo de desarrollar preeclampsia por la importante carga económica mundial que demanda esta enfermedad.

\section{PALABRAS CLAVES: Hipertensión arterial, preeclampsia, eclampsia, salud pública, mortalidad materna, mortalidad perinatal}

\section{SUMMARY}

Preeclampsia is a public health problem and a major cause of maternal death. The prevalence ranges from $1.8-16.7 \%$, but the cause remains unknown and is associated with significant health problems, many challenges exist for the prediction, prevention and treatment, prophylactic measures such as low-dose aspirin and calcium supplements still require more evidence for routine use, prenatal care, timely diagnosis, proper management, and childbirth are the most effective measures to reduce mortality from this cause. Social factors and prompt medical attention should prevail in the population, but these are limited in underserved populations without access to medical services. We can conclude that the health systems of all populations to identify and medically assist women who are at greater risk of developing preeclampsia by the global economic toll this disease demand.

\section{KEY WORDS: Hypertension, preeclampsia, eclampsia, public health, maternal mortality,} perinatal mortality 


\section{INTRODUCCIÓN}

La preeclampsia es un trastorno hipertensivo inducido por el embarazo que se manifiesta clínicamente después de las 20 semanas de gestación. Fue descrita hace más de 2 mil años; la falta de un manejo oportuno, conduce a eclampsia $(1,2)$, sin embargo, la causa sigue desconocida y se asocia a problemas de salud materna-perinatal importantes. La preeclampsia y eclampsia se manifiestan clínicamente con todo un espectro de síntomas clínicos por la misma condición. En la preeclampsia están presentes principalmente la hipertensión y proteinuria, además de edema, cuando se presentan convulsiones, además de estos síntomas, se diagnostica como eclampsia (1-3).

La preeclampsia constituye un problema de salud pública porque incide significativamente en las tasas de morbilidad y mortalidad materna perinatal a nivel mundial (3-5). Sin embargo, el impacto de la enfermedad es más severo en países en desarrollo $(6,7)$, donde otras causas también frecuentes, ocasionan mortalidad materna (por ejemplo, hemorragia y sepsis). El manejo médico es ineficaz debido a la presentación tardía de la enfermedad $(8,9)$; el problema se complica por su etiología y naturaleza impredecible (10), que hace necesario contar con un control efectivo de la preeclampsia y sugerir las medidas para aplicarlas en cualquier parte de la población. La presente revisión de la literatura proporciona evidencia sobre el diagnóstico, prevención, tratamiento de la preeclampsia.

\section{EPIDEMIOLOGÍA DE LA PREECLAMPSIA}

A nivel mundial, la incidencia de preeclampsia oscila entre $2-10 \%$ de los embarazos, la cual es precursor de la eclampsia y varía en todo el mundo. La Organización Mundial de la Salud (OMS) estima que la incidencia de preeclampsia es siete veces mayor en los países en desarrollo que en los desarrollados $(2,8 \%$ y $0,4 \%$ de los nacidos vivos respectivamente). La incidencia de eclampsia en los países desarrollados de Norteamérica y Europa es similar y se estima alrededor de 5 a 7 casos por cada 10.000 partos, mientras que en países en desarrollo es variable, oscilando entre 1 caso por cada 100 embarazos a 1 por cada 1.700 embarazos (1-3). Las tasas de los países africanos como Sudáfrica, Egipto, Tanzania y Etiopía varían de $1,8 \%$ a $7,1 \%$ y en Nigeria, la prevalencia oscila entre $2 \%$ a $16,7 \%(11)$.

\section{ALGUNOS ASPECTOS DE LA PREECLAMPSIA EN SALUD PÚBLICA}

Los retos en la prevención de la preeclampsia, como cualquier proceso de otras enfermedades requiere la disponibilidad de métodos para la predicción de aquellas con mayor riesgo de desarrollar el trastorno. Aunque numerosos ensayos clínicos y bioquímicos se han propuesto para la predicción o la detección temprana de la preeclampsia, la mayoría siguen siendo poco realistas para su uso general en la mayoría de los casos. En la actualidad, no existe una sola prueba de detección fiable y rentable para la preeclampsia, que pueda ser recomendada para su uso rutinario (12). Aunque algunos estudios para la detección temprana de la preeclampsia como los estudios Doppler sobre la arteria uterina en el primer trimestre del embarazo y los marcadores séricos maternos se han mostrado prometedores $(13,14)$ pero no existe evidencia suficiente para sugerir su uso rutinario en la práctica clínica (15).

En relación a la prevención, el manejo terapéutico con aspirina ha demostrado ser beneficioso en la disminución de la incidencia de preeclampsia en poblaciones específicas, por ejemplo, los que tienen el flujo Doppler uterino anormal en el segundo trimestre (16-19), pero no se recomienda su uso generalizado en todos los pacientes y no está basado en la evidencia. En el mismo sentido, a pesar de la revisión Cochrane, se ha manifestado una cierta ventaja en los suplementos de calcio, en particular para los grupos de mayor riesgo y aquellos con una baja ingesta de calcio (20), pero el problema de la selección de pacientes adecuados para el inicio del tratamiento puede ser una carga desde una perspectiva de salud pública. Del mismo modo, los hallazgos de estudios anteriores que manifestaron beneficios con la suplementación de vitaminas (2125) han sido refutadas por un estudio reciente de la OMS, en particular las vitaminas C y E (26).

Los desafíos en el manejo de la preeclampsia, para hacer frente a ésta en cualquier población, consideran que los sistemas de salud sean eficaces y de fácil acceso a la atención de la salud. Sin embargo, en la gran mayoría de las poblaciones marginadas, el acceso a los servicios de salud es limitado, por una serie de factores: retraso en la toma de decisión para buscar asistencia médica, por falta de información sobre hospitales donde acudir. Esto se agrava por la falta de decisión, pobreza y costo de la atención médica, cuando no existe seguridad social de la población que ocasiona y suele dar lugar a retrasos 
fatales en la búsqueda de atención profesional (27), en particular por aspectos socio-económicos, como nivel de educación, estado civil y culturales de la madre (28).

La falta de acceso a una atención médica de calidad es el principal obstáculo para la reducción de la mortalidad materna (27), debido al lugar, distancia y falta de transporte a los hospitales de especialidad. En algunos lugares del mundo el $50 \%$ de las mujeres rurales viven a más de $5 \mathrm{~km}$ del hospital más cercano, y muchas no tienen manera de llegar por falta de la distribución equitativa en los establecimientos de salud que están a favor de las zonas urbanas. Además, de los tratamientos de medicina alternativa y tradicional de estas zonas que impiden el envío de estas mujeres a servicios de salud y que ocasionan $46,4 \%$ de los casos de preeclampsia (29).

Los retrasos en la prestación de atención médica, también contribuyen a la mortalidad materna, por falta de atención medica que necesitan antes, durante y después del parto, particularmente en situaciones de emergencia, incluso las actitudes y calidad de atención por los médicos a poblaciones marginadas también se identifican como barreras, esto se agrava por la falta de personal capacitado, equipos y suministros. Por ejemplo, a pesar de que la eficacia del sulfato de magnesio ha sido documentada por varios investigadores, los estudios han demostrado que el sulfato de magnesio no se administra rutinariamente (30), y su uso se limita a los hospitales de enseñanza. La falta de disponibilidad de los medicamentos, personal de salud apropiados necesarios para su administración y costo son los obstáculos que suelen plantearse.

\section{MODELOS DE ATENCION PÚBLICA}

En los diferentes niveles del sistema de salud tanto macro y micro, existen normas incorrectas para su implementación y toma de decisiones. Por ejemplo, datos estadísticos fiables sobre las mujeres que mueren por eclampsia son difíciles de obtener debido a la mala calidad de los sistemas de registro de las estadísticas vitales y registros hospitalarios, en zonas marginadas, donde un número considerable de partos tiene lugar en casa y por lo tanto, no existen registros para estos nacimientos y sus secuelas. Además, las políticas de salud por lo general no están basadas en la evidencia, por la falta de información.

\section{RECOMENDACIONES}

Al evaluar el manejo clínico, la OMS se centra en la estrategia de la atención prenatal recomendando la detección de la preeclampsia durante la tercera visita prenatal a las 32 semanas, principalmente en zonas desprotegidas donde se debe seguir basando en antecedentes obstétricos, médicos y examen clínico de las mujeres. Las mujeres embarazadas deben ser evaluadas en su primera consulta prenatal sobre los factores de riesgo de preeclampsia, como edad joven, nuliparidad, primer embarazo después de los 35 años, obesidad previa al embarazo actual, embarazo múltiple, historia previa de preeclampsia, diabetes mellitus e hipertensión arterial sistémica (1-3,31-33); sin embargo, es importante señalar que la presencia de estos factores no son fiables para desarrollar preeclampsia y se ha demostrado que la detección materna para la preeclampsia con una historia clínica correcta es de sólo $45,3 \%$ de los casos (34).

Los exámenes rutinarios para preeclampsia con relación a la toma de la presión arterial en todas las mujeres embarazadas deben practicarse a acuerdo a lo recomendado por la OMS (35). Cuando los recursos están disponibles, lo mejor es medir la presión arterial con un esfigmomanómetro de mercurio. Un análisis de orina para proteinuria también debe realizarse rutinariamente en cada visita prenatal en todos los servicios de salud, como complemento a la medición de rutina de la presión arterial. Los criterios para el diagnóstico de preeclampsia desarrollados por el Grupo de Presión Arterial Programa Nacional de Educación de Trabajo, que todavía se utilizan tradicionalmente en la práctica clínica como son: presión arterial sistólica mayor o igual a $140 \mathrm{mmHg}$ o presión arterial diastólica mayor o igual a $90 \mathrm{mmHg}$, en por lo menos dos ocasiones con un intervalo de 4 a 6 horas de diferencia, que se presenta después de 20 semanas de gestación en una mujer cuya presión arterial había sido normal. Además de esto, contribuye al diagnóstico la presencia de proteinuria mayor o igual a 0,3 gramos en una muestra de orina de 24 horas o su equivalente en tiras reactivas (más de 1+) en dos muestras de orina al azar recolectadas con cuatro o más horas de separación $(36,37)$.

Una vez reconocida la preeclampsia y dependiendo de la gravedad, las opciones de cuidado incluyen evaluación continua materno-fetal, tratamiento anti-hipertensivo e inducción del parto (único tratamiento curativo). Existe evidencia significativa que el uso de sulfato de magnesio previene y trata las convulsiones en mujeres con preeclampsia severa y eclampsia $(1-3,36,38)$ comparado con diazepam, fenitoína y cóctel lítico, también redujo la tasa de mortalidad materna y recurrencia de las convulsiones (39), sin embargo, es necesario garantizar la atención postparto, durante el parto y 
puerperio, por profesional calificado para prevenir y tratar las convulsiones. Las visitas postnatales son importantes para evaluar la recuperación de la mujer y discutir los riesgo para la salud materna futura.

\section{ASPECTOS SOCIALES DE LA PREECLAMPSIA}

Los factores sociales influyen en la preeclampsia con $27 \%$ de las muertes maternas y es importante aumentar la conciencia en las mujeres sobre la necesidad de recibir atención de emergencia, si surgen complicaciones durante el parto, para asegurar la intervención médica rápida, eficaz e incrementar la probabilidad de éxito terapéutico (13). Toda la comunidad debe ser capacitada para reconocer los signos, desarrollar planes en situaciones de emergencia e incluir el traslado a hospitales, porque la reducción del riesgo de muerte se vuelve más difícil cuando se han desarrollado complicaciones.

\section{SISTEMAS DE SALUD}

La mayoría de las muertes maternas durante el parto se producen por el mal desempeño de la asistencia médica (40). Para reducir la mortalidad materna $(41,42)$ la seguridad de la atención de la madre debe ser un problema prioritario de salud, por los gobiernos y personal de salud, con énfasis en la calidad y rendición de cuentas, también necesarias para asegurar la confianza de las pacientes (43). El compromiso de los sistemas de salud es contar con recursos necesarios en el sector salud, para mejorar la calidad de los servicios obstétricos de emergencia, que garanticen la disponibilidad de personal capacitado, medicamentos y equipos en todos los niveles de atención. Servicios de referencia para atención obstétrica de emergencia rápidos y económicos, para limitar los retrasos en su atención (44).

Las mejoras en la prestación de servicios se pueden lograr mediante protocolos o guías de prácticas clínicas rápidas, para el manejo de casos de emergencias obstétricas en cada nivel de atención y seguimiento de su aplicación. El sulfato de magnesio debe ser parte de la lista de medicamentos esenciales en todas partes como tratamiento de primera línea para la preeclampsia y eclampsia. Para mejorar la salud materna, las barreras de acceso a los servicios de salud deben ser identificadas y abordadas en todos los niveles (45).

El uso de datos en particular para mejorar los sistemas de notificación y registro para estimar la carga de la enfermedad facilitará la planificación y prestación de los servicios (42). Las auditorías de muerte materna ayudarían a la comprensión de las causas de la preeclampsia y eclampsia, a evitar la muerte e identificar la atención deficiente y factores evitables, relacionados con el fallecimiento materno (46).

\section{CONCLUSIONES}

La preeclampsia y eclampsia debe ser identificada como un problema prioritario para reducir la mortalidad materna en todos los países, principalmente los marginados, fortaleciendo los sistemas de salud pública y mejorando el acceso de la madre a personal de salud capacitado. Se necesitan más investigaciones para comprender las causas y mejorar las estrategias preventivas. El mejor acceso a la atención obstétrica adecuada, en particular durante el parto, y mejor detección y tratamiento de los casos identificados, reducirá los índices de morbimortalidad materna y perinatal.

\section{REFERENCIAS}

1. Vargas-Hernández VM. Enfermedad hipertensiva durante el embarazo. Rev Hosp Jua Mex 1999;6:125-34.

2. Myers JE, Baker PN. Hypertensive diseases and eclampsia. Curr Opin Obstet Gynecol 2002;14:119-25.

3. World Health Organization. Global Program to Conquer Preeclampsia/Eclampsia. 2002.

4. Shah A, Fawole B, M'Imunya JM. Cesarean delivery outcomes from the WHO global survey on maternal and perinatal health in Africa. Intl J Gynec Obstet 2009;107:191-7.

5. McClure EM, Saleem S, Pasha O, Goldenberg RL. Stillbirth in developing countries: a review of causes, risk factors and prevention strategies. J Matern Fetal Neonatal Med 2009:22:183-90.

6. Igberase G, Ebeigbe P. Eclampsia: ten-years of experience in a rural tertiary hospital in the Niger delta, Nigeria. J Obstet Gynaecol 2006;26:414-7.

7. Adamu YM, Salihu HM, Sathiakumar N, Alexander GR. Maternal mortality in Northern Nigeria: a population-based study. Eur J Obstet Gynecol Reprod Biol 2003;1098:153-9.

8. Ikechebelu JI, Okoli CC. Review of eclampsia at the Nnamdi Azikiwe University teaching hospital, Nnewi (January 1996-December 2000. J Obstet Gynaecol 2002;22:287-90.

9. Onuh SO, Aisien AO. Maternal and fetal outcome in eclamptic patients in Benin City, Nigeria. J Obstet Gynaecol 2004;24:765-8.

10. Duley L. Pre-eclampsia and the hypertensive disorders of pregnancy. Br Med Bull 2003;67:161-76.

11. Thiam M, Goumbala M, Gning SB, Fall PD, Cellier C, Perret JL. Maternal and fetal prognosis of hypertension and pregnancy in Africa (Senegal). J Gynecol Obstet Biol Reprod 2003;32:35-8.

12. Wagner LK, Diagnosis and management of preeclampsia. Am Fam Phys 2004;70:2317-24.

13. Kharb $S$. Serum markers in pre-eclampsia. Biomarkers 2009;14: 395-400. 
14. Audibert F, Boucoiran I, An N, Aleksandrov N, Delvin E, Bujold E, Rey E. Screening for preeclampsia using first-trimester serum markers and uterine artery Doppler in nulliparous women. Am J Obstet Gynecol 2010;203:383.e1-8.

15. Papageorghiou AT, Campbell S. First trimester screening for preeclampsia. Current Opin Obstet Gynecol 2006;18:594-600.

16. Louden k, Kilby M. Low-dose aspirin: the rationale for preventing pre-eclampsia and intra-uterine growth retardation: a role after CLASP. In: Recent Advances in Obstetrics and Gynaecology, 1995;Num 19, Bonnar J, Ed., Churchill Livingstone, Edinburgh, UK, 1995.

17. Bujold E, Roberge S, Lacasse Y. Prevention of preeclampsia and intrauterine growth restriction with aspirin started in early pregnancy: a meta-analysis. Obstet Gynecol 2010;116:402-14.

18. Knight M, Duley L, Henderson-Smart DJ, King JF. Antiplatelet agents for preventing and treating pre-eclampsia. Cochrane Database of Syst Rev 2000;(2):CD000492.

19. Hofmeyr GJ, Lawrie TA, Atallah AN, Duley L. Calcium supplementation during pregnancy for preventing hypertensive disorders and related problems. Cochrane Database Syst Rev. 2006 Jul 19;(3):CD001059.

20. Bodnar LM, Catov JM, Simhan HN, Holick MF, Powers RW, Roberts JM, Maternal vitamin D deficiency increases the risk of preeclampsia. J Clin Endocrinol Metabol 2007;92:3517-22.

21. Bodnar LM, Tang G, Ness RB, Harger G, Roberts JM. Periconceptional multivitamin use reduces the risk of preeclampsia. Am J Epidemiol 2006;164:470-7.

22. Roberts JM, Balk JL, Bodnar LM, Belizán JM, Bergel E, Martinez A. Nutrient involvement in preeclampsia. J Nutr 2003;133(5 Suppl 2):1684S-92S.

23. Wen SW, Chen XIK, Rodger M. Folic acid supplementation in early second trimester and the risk of preeclampsia. Am J Obstet Gynecol 2008;198:45.e1-45.e7.

24. Chappell LC, Seed PT, Briley AL .Effect of antioxidants on the occurrence of pre-eclampsia in women at increased risk: a randomised trial. Lancet 1999;354:810-6.

25. Villar J, Purwar M, Merialdi M . World Health Organization multicentre randomised trial of supplementation with vitamins $C$ and $E$ among pregnant women at high risk for pre-eclampsia in populations of low nutritional status from developing countries. BJOG 2009;116:780-8.

26. Richard F, Witter S, De Brouwere V. Innovative approaches to reducing financial barriers to obstetric care in low-income countries. Am J Public Health 2010;100:1845-52.

27. Olusanya BO, Alakija OP, Inem VA. Non-uptake of facility-based maternity services in an inner-city community in Lagos, Nigeria: an observational study. J Biosocial Scie 2010;42:341-58.

28. Onah HE, Okaro JM, Umeh U, Chigbu CO. Maternal mortality in health institutions with emergency obstetric care facilities in Enugu State, Nigeria. J Obstet Gynaecol 2005;25:569-74.

29. Diaz C. Magnesium sulfate use for the treatment of severe preeclampsia and eclampsia in Mexico. In:
Proceedings of the Global Maternal Health Conference, New Delhi, India. 2010.

30. Conde-Agudelo A, Belizán JM. Risk factors for preeclampsia in a large cohort of Latin American and Caribbean women. Brit J Obstet Gynaecol 2000;107:7583.

31. Magnussen EB, Vatten LJ, Lund-Nilsen TI, Salvesen KÅ, Smith GD, Romundstad PR. Prepregnancy cardiovascular risk factors as predictors of preeclampsia: population based cohort study. Br Med $\mathrm{J}$ 2000;335:978-81.

32. Packer CS. Biochemical markers and physiological parameters as indices for identifying patients at risk of developing pre-eclampsia. J Hypertens 2005;23:45-6.

33. Papageorghiou AT, Yu CKH, Erasmus IE, Cuckle HS, Nicolaides $\mathrm{KH}$. Assessment of risk for the development of pre-eclampsia by maternal characteristics and uterine artery Doppler. BJOG 2005;112:703-9.

34. WHO, UNICEF. Antenatal Care in Developing Countries: Promises, Achievements and Missed Opportunities. Hallado en: http://www.childinfo.org/files/antenatal_care.pdf

35. Schroeder BM. ACOG practice bulletin on diagnosing and managing preeclampsia and eclampsia. American Family Physician, 2002;66(2):330-1.

36. Duley L. Pre-eclampsia, eclampsia, and hypertension. Clinic Evidence, 2008;2008:1402.

37. Duley L. Do women with pre-eclampsia, and their babies, benefit from magnesium sulphate? The Magpie Trial: a randomised placebo-controlled trial. Lancet 2002;359:1877-90.

38. Aaserud M, Lewin S, Innvaer S, Paulsen EJ, Dahlgren AT, Trommald M, Duley L, Zwarenstein M, Oxman AD. Translating research into policy and practice in developing countries: a case study of magnesium sulphate for pre-eclampsia. BMC Health Serv Res 2005;5:68.

39. Lawn JE, Kinney M, Lee AC. Reducing intrapartumrelated deaths and disability: can the health system deliver? Int J Gynaecol Obstet 2009;107:S123-S140.

40. Lim SS, Dandona L, Hoisington JA, James SL, Hogan MC, Gakidou E. India's Janani Suraksha Yojana, a conditional cash transfer programme to increase births in health facilities: an impact evaluation. Lancet 2010;375:2009-23.

41. Prata N, Passano P, Sreenivas A, Gerdts CE. Maternal mortality in developing countries: challenges in scaling-up priority interventions. Women's Health 2010;6:311-27.

42. Olsen OE. The impact of global health initiatives on trust in health care provision under extreme resource scarcity: presenting an agenda for debate from a case study of emergency obstetric care in Northern Tanzania. Health Res Policy Sys 2010;8:14.

43. Ijadunola KT, Ijadunola MY, Esimai OA, Abiona TC. New paradigm old thinking: The case for emergency obstetric care in the prevention of maternal mortality in Nigeria. BMC Women's Health 2010;10:6.

44. Lee AC, Lawn JE, Cousens S. Linking families and facilities for care at birth: what works to avert intrapartum-related deaths? Inter J Gynaecol Obstet 2009;107:S65-S86. 
45. Aagaard-Tillery KM, Belfort MA. Eclampsia: Morbidity, Mortalily, and Management, Clin Obstet Gynecol 2005;48:13-23.

46. Sorensen BL, Elsass P, Nielsen BB, Massawe S,
Nyakina J, Rasch V. Substandard emergency obstetric care a confidential enquiry into maternal deaths at a regional hospital in Tanzania. Trop Med Inter Health 2010;15:894-900. 